\title{
Micellar Effects on Nucleophilic Addition Reaction and Applicability of Enzyme Catalysis Model
}

\author{
R. K. LONDON SINGH \\ Department of Chemistry, D.M. College of Science \\ Imphal-795001, India \\ london_ningthemcha@yahoo.com
}

Received 4 July 2011; Accepted 20 September 2011

\begin{abstract}
This study describes the effect of anionic and cationic micelles on nucleophilic addition reaction of rosaniline hydrochloride $(\mathrm{RH})$ with hydroxide under pseudo-first order condition. Strong inhibitory effect is observed due to SDS micelle, whereas CTAB catalysed the reaction. This is explained on the basis of electrostatic and hydrophobic interactions which are simultaneously operating in the reaction system. The kinetic data obtained is quantitatively analysed by applying the positive cooperativity model of enzyme catalysis. Binding constants and influence of counterions on the reaction have also been investigated.
\end{abstract}

Keywords: Micelles, Positive cooperativity, Triphenylmethane dyes.

\section{Introduction}

Due to ability in providing catalytic interfaces, many physico-chemical properties are affected in micellar system, thus leading to various analytical, biological, chemical, and industrial applications. So, investigation of chemical reaction involving micelle has been of immense interest for many workers ${ }^{1-7}$. It has also been shown by many workers that chemical reactions in micellar system are analogous to enzymatic reactions in biological systems by citing various types of chemical reactions in micellar medium. Thus, chemical reactions in micellar system serve as simple model for many biological processes which occur at or involve amphipathic surfaces ${ }^{5,7}$. Studies of some of triphenylmethane dyes in micellar medium have been reported, but so far exhaustive studies of kinetics of nucleophilic addition reaction of rosaniline hydrochloride ( $\mathrm{RH})$ a triphenylmethane dye which is very important for bacteriology ${ }^{8}$ is very rare. The carbonium ions are frequently encountered in several organic and enzymatic reactions and therefore, studies involving carbonium ions in micellar media are of great importance ${ }^{9,10}$.

The present paper reports the effect of anionic surfactant sodiumdodecyl sulphate (SDS) and cationic surfactant cetyltrimethylammonium bromide (CTAB) on the reaction of rosaniline hydrochloride $(\mathrm{RH})$ carbonium ion with hydroxide ion. Analysis and quantitative treatment of experimental data have been done successfully by applying positive cooperativity model 
of enzyme catalysis. The effect of counterions on the reaction system has been investigated and the result closely parallels the lyotropic series. The observed catalysis and inhibition have been explained on the basis of both hydrophobic and hydrophilic interactions in catalytic micellar aggregates of various species present in the reaction system. The values of binding constants of substrate with micellar aggregates have been calculated. The structure of rosaniline hydrochloride carbonium ion and its reaction with $\mathrm{OH}^{-}$is given in Figure 1.

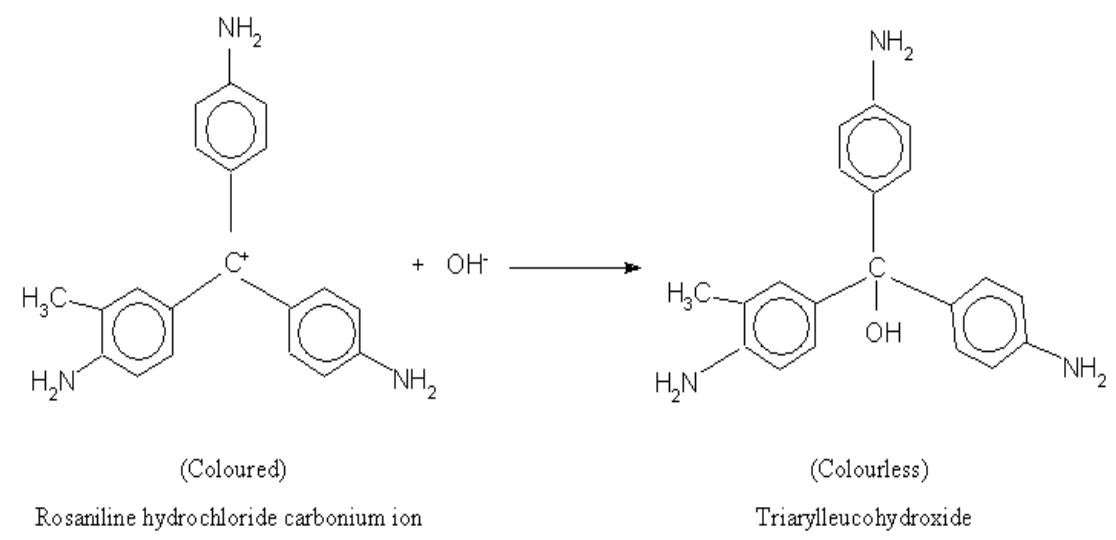

Figure 1 Reaction of Rosaniline hydrochloride carbonium ion with $\mathrm{OH}^{-}$.

\section{Experimental}

The cationic surfactant (CTAB) was obtained from SISCO Chemical Industries, India. The anionic surfactant (SDS) and Rosaniline hydrochloride (RH) dye were obtained from Central Drug House, India. Sodium hydroxide and Sodium bromide were from Loba Chemical Industries. Sodium fluoride and Sodium chloride were obtained from Galxo Laboratories, India. All the reagents used were of analytical grade. The solutions were prepared in double- distilled water. All the reactions were carried out by following decolourization reaction using Beckmann DU-6 Spectrophotometer in visible range at $25^{0} \pm 0.1^{\circ} \mathrm{C}$ using a high precision thermostat. The reactions follow pseudo-first order kinetics and were monitored by observing the change in absorbance as a function of time at absorption maxima $(556 \mathrm{~nm})$ in time drive mode. Slopes and rate constants were calculated from the straight line plots by linear regression method using a personal computer.

\section{Results and Discussion}

The visible absorption maxima $545 \mathrm{~nm}$ (in aqueous media in absence of surfactant) of rosaniline hydrochloride $(\mathrm{RH})$ exhibited a red shift in surfactant solutions (0.02 M CTAB and $0.005 \mathrm{M} \mathrm{SDS}$ ) to $556 \mathrm{~nm}$. This bathochromic shift evidenced binding of substrate (dye carbonium ion $\mathrm{RH}^{+}$) with micelles. It has been observed that SDS strongly inhibits the reaction of $\mathrm{OH}^{-}$with $\mathrm{RH}^{+}$with overall inhibition factor $k_{r e l}{ }^{-}\left(=k_{w} / k_{m}\right)$ being 89 where, $k_{w}$ is rate constant in aqueous phase and $k_{m}$ is rate constant in micellar phase. The rate constant versus SDS concentration profile (at $\left[\mathrm{OH}^{-}\right]=1.85 \times 10^{-2} \mathrm{M}$ and $\left[\mathrm{RH}^{+}\right]=3.75 \times 10^{-5} \mathrm{M}$ ) is shown in Figure 2(A) whereas, CTAB catalyses the reaction and variation of pseudo-first order rate constant as a function of CTAB concentration (at $\left[\mathrm{OH}^{-}\right]=1.85 \times 10^{-3} \mathrm{M}$ and $\left.\left[\mathrm{RH}^{+}\right]=3.75 \times 10^{-5} \mathrm{M}\right)$ is shown in Figure 2(B). 


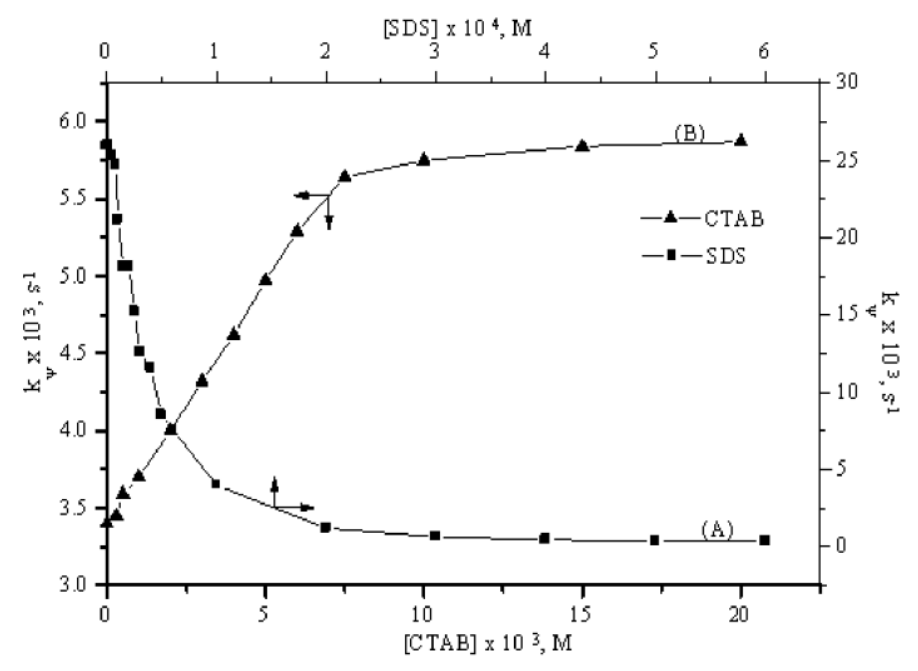

Figure 2 Dependence of the rate constant $\left(k_{\psi}\right)$ on surfactant concentration. (A): SDS inhibited reaction of $\mathrm{RH}^{+}$with $\mathrm{OH}^{-}$; (B): CTAB catalysed reaction of $\mathrm{RH}^{+}$with $\mathrm{OH}^{-}$.

The overall catalysis factor $k_{r e l}{ }^{+}\left(=k_{m} / k_{w}\right)$ for $\mathrm{RH}^{+}$was found to be 1.72 . From the experimental data the kinetic $\mathrm{CMC}$ values were found to be $7.5 \times 10^{-3} \mathrm{M}$ for CTAB and $1 \times 10^{-5} \mathrm{M}$ for SDS micelles.

The rate constant is found to be independent of dye (substrate) concentration at fixed surfactant and $\mathrm{OH}^{-}$concentration and it increases linearly with increasing $\left[\mathrm{OH}^{-}\right]$at fix surfactant and dye concentrations. This shows that the reaction mechanism remained pseudo-first order in presence of micelle.

The inhibitory effect on rate due to SDS micelles can be explained by considering both electrostatic and hydrophobic interactions which operates simultaneously in the reaction system ${ }^{11}$. In presence of SDS micelles, as $\mathrm{OH}^{-}$bears similar charge as that of anionic micelles, so they are unable to approach the strongly bound substrate with negatively charged SDS micelles as effectively as it can do in absence of SDS micelles, thus exhibiting inhibitory effect. But, in presence of $\mathrm{CTAB}$, hydroxide ion being negatively charged has favorable interaction with positively charged $\mathrm{CTAB}$ micellar aggregates containing hydrophobic substrate. Thus, the approach of $\mathrm{OH}^{-}$is facilitated towards substrate and local concentration of $\mathrm{OH}^{-}$increases around the micellar aggregates, leading to catalytic effect by CTAB.

A kinetic model developed by Piszkiewicz ${ }^{14,15}$ has been used for quantitative analysis of micellar data. This model is analogous to Hill model which describes the positive cooperativity in enzyme catalysis. Thus, by analogy, with reference to micellar catalysis, cooperativity is defined as stimulation of association of additional surfactant molecules to an aggregate as a result of interaction of the first surfactant molecule.

Piszkiewicz's model assumes that a substrate, $S$ (dye carbonium ion in this case) and $n$, number of surfactant (detergent) molecules $D$, aggregate to form catalytically functional micellar aggregate $\left(D_{n} S\right)$, which may undergo reaction to yield product by following Scheme 1: 


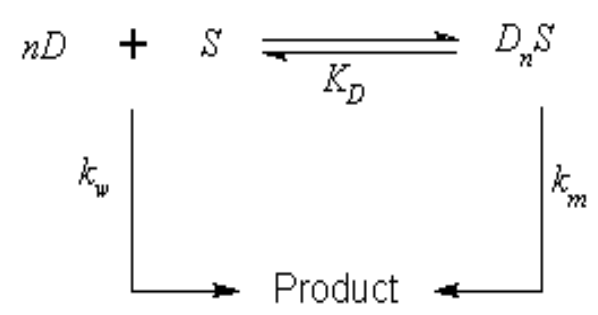

Scheme 1.

$K_{D}$ represents dissociation constant of micelle (its reciprocal is binding constant), $k_{m}$ and $k_{w}$ are rate constants of the reaction in micellar and bulk (aqueous) phases. For this reaction scheme, pseudo-first order rate constant $k_{\Psi}$ is expressed as a function of concentration of detergent $D$, by the equation

$$
k_{\psi}=\frac{k_{m}[D]^{n}+k_{w} K_{D}}{K_{D}+[D]^{n}}
$$

Which can be written in the form,

$$
\log \frac{k_{\Psi}-k_{w}}{k_{m}-k_{\Psi}}=n \log [D]-\log K_{D}
$$

The point of half maximal catalysis or inhibition designated as $[\mathrm{D}]_{50}$ or $\log [\mathrm{D}]_{50}$ can be obtained when the left hand side of equation (2) equals zero.

Thus,

$$
\log [D]_{50}=\frac{\log K_{D}}{n}
$$

In this model, slope $(n)$ of double log plot describes the stoichiometry of the reaction scheme and it is considered as an index of cooperativity in enzymatic reactions. Thus, index of cooperativity is a sensitive measure of interaction between substrate and surfactant. The value of $n>1$ reflects positive cooperativity. The calculated binding constant according to the above model, substrate with anionic surfactant SDS $\left(7.9 \times 10^{5}\right)$ is much more than cationic surfactant CTAB $\left(3.1 \times 10^{2}\right)$ which is in accordance with the existing electrostatics and hydrophobic interaction in the reaction system (both interactions are favorable for SDS, but only hydrophobic interaction is favourable for $\mathrm{CTAB})$. Also, $[D]_{50}$ values for $\operatorname{SDS}\left(2.88 \times 10^{-5} \mathrm{M}\right)$ as compared to CTAB $\left(3.89 \times 10^{-3} \mathrm{M}\right)$ supports stronger binding of substrate to those micelles having favorable electrostatics-hydrophobic environment.

The applicability of equation (2) was tested using the micellar data of Figure 2(A) for SDS and Figure 2(B) for CTAB. The Hill type double log plots and the quantitative analysis according to equation (2) and (3), for the reaction under study is shown in Figure 3 and Table 1 respectively. 
Table 1. Piszkiewicz's model derived parameters for CTAB and SDS influenced reactions of Rosaniline hydrochloride carbonium ion $\left(\mathrm{RH}^{+}\right)$with $\mathrm{OH}^{-}$at $25^{0} \pm 0.1^{0} \mathrm{C}$.

\begin{tabular}{|c|c|c|c|c|c|}
\hline Micelles & 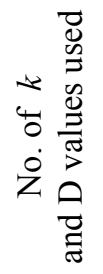 & $\begin{array}{l}\text { Slope ( index of } \\
\text { cooperativity) }(n)\end{array}$ & {$[D]_{50,} \mathrm{M}$} & $\begin{array}{l}\text { Intercept } \\
\left(-\log K_{D}\right)\end{array}$ & $\begin{array}{c}\text { Binding } \\
\text { constant } \\
\left(1 / K_{D}\right)\end{array}$ \\
\hline SDS & 5 & 1.3 & $2.88 \times 10^{-5}$ & 5.9 & $7.9 \times 10^{5}$ \\
\hline CTAB & 4 & 1.07 & $3.89 \times 10^{-3}$ & 2.5 & $3.1 \times 10^{2}$ \\
\hline
\end{tabular}

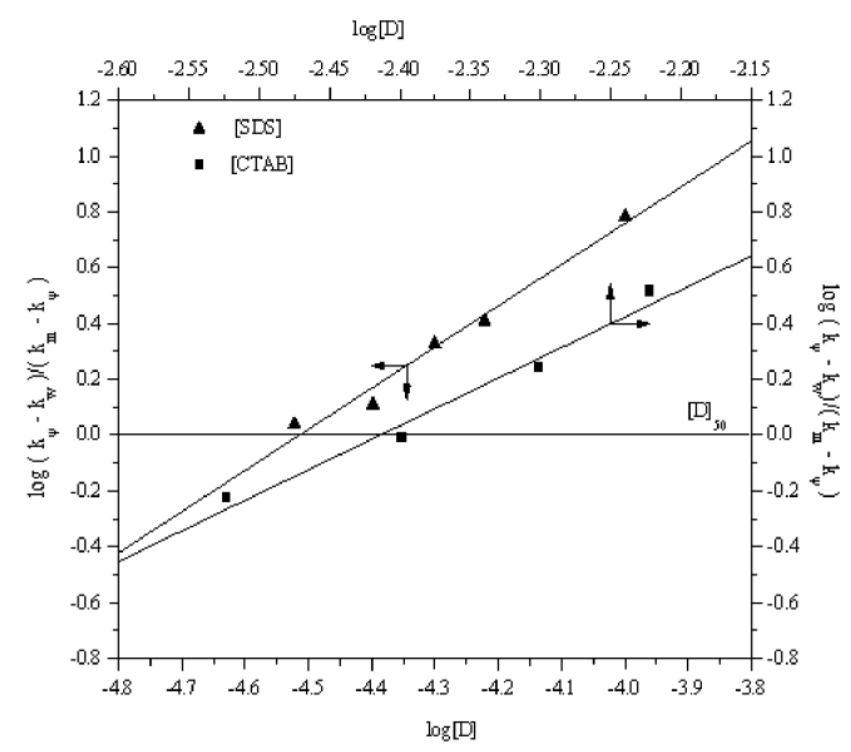

Figure 3. Quantitative treatment of the effect of surfactants CTAB and SDS on the reaction of $\mathrm{RH}^{+}$with $\mathrm{OH}^{-}$, applying Positive Cooperativity Model of Enzyme catalysis (Hill type plots).

It is to note that the value of $n$ found was greater than 1 evidencing for positive cooperativity in the reaction system ${ }^{12,13}$. The effect of added counterions (ions opposite to the charge on the micellar surface) on the CTAB catalysed reaction of $\mathrm{RH}^{+}$with $\mathrm{OH}^{-}$has been investigated by varying concentration of certain chosen electrolytes. All the added counterions $\left(\mathrm{F}^{-}, \mathrm{Cl}^{-}\right.$, and $\left.\mathrm{Br}^{-}\right)$exhibited inhibitory effect on reaction rates in presence of CTAB. The added counterions reduced the extent of overall catalysis caused by CTAB micelles as shown in Fig. 4 in the order: $\mathrm{Br}^{-}>\mathrm{Cl}^{-}>\mathrm{F}^{-}$and is suggested that counterions with low charge density have more pronounced inhibitory effect. The result closely parallels the lyotropic series ${ }^{16}$ which represents salt effect on precipitation and denaturation of proteins. 


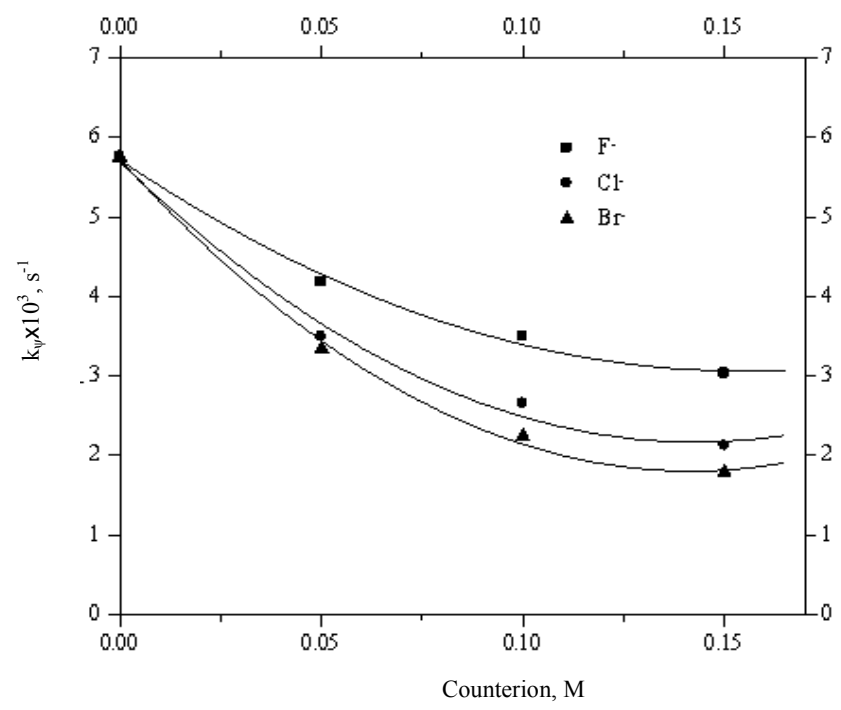

Figure 4. Effect of added counterions on the rate constant for CTAB catalysed reaction of $\mathrm{RH}^{+}$with $\mathrm{OH}^{-}$.

\section{Conclusion}

From the studies it is concluded that the nucleophilic addition reaction of rosaniline hydrochloride with hydroxide is catalysed by cationic micelle, CTAB and inhibited by anionic micelle, SDS. The observed catalysis and inhibition have been attributed to both hydrophobic and electrostatic interactions in catalytic micellar aggregates of various species. The positive cooperativity in enzyme catalysis has been tested and found applicable in the reaction system. All the added counterions exhibited inhibitory effect on reaction rate in presence of CTAB micelles and they are in the order of $\mathrm{Br}^{-}>\mathrm{Cl}^{-}>\mathrm{F}^{-}$, which shows that the counterions with low charge density have more pronounced inhibitory effects.

\section{References}

1. Bunton C A, Nome F, Quina F H and Romsted L R, Acc Chem Res., 1991, 24, 357.

2. Stamouli A, Pelletier E, Koustos V, Vander Vegte E and Hadzuoannou G, Langmuir, 1996, 12, 13221.

3. Frindi M, Michels B and Zana R, J Phys Chem., 1992, 96, 6095.

4. Gratzel M and Kalyansundaram K, Kinetics and Catalysis in Microheterogeneous System; Marcel Dekker: New York, 1991.

5. Fendler J H, Membrane Mimetic Chemistry; John Wiley: New York, 1982.

6. Mittal K L, Surfactants in Solution, Vol. 7-10, Plenum, New York, 1989.

7. Holmberg K, Jonsson B, Kronberg B and Lindman B, Surfactants and Polymers in Aqueous Solution; John Wiley and Sons: West Sussex, 2003.

8. Goyal. S.K, J Environ Monit., 2001, 3, 666-670.

9. Albrizzio A J, Archilla J, Rodulfo T and Cordes E H, J Org Chem., 1972, 37, 871.

10. Funasaki N, J Colloid Interface Sci., 1977, 62, 336.

11. Adina Raducan, Alexandra Olteanu, Mihaela Puiu, Dumitru Oancea, Cent Eur J Chem., 2008, 6 (1), 89-92.

12. Babak Samiey and Ali Raoof Toosi, Bull Korean Chem Soc., 2009, 30(9), 2051-2056. 
13. Owoyomi Olanrewaju, Jide Ige, Olandega Soriyan, Ogunlusi Grace, Olaseni Segun Esan and Oladimeji Olanrewaju, Acta Chim Slov., 2007, 54, 370-374.

14. Piszkiewicz D, J Am Chem., 1976, 98, 3053.

15. Piszkiewicz D, J Am Chem., 1977, 99, 1550, 7695.

16. Ray A and Nemethy G, J Am Chem Soc.,s 1971, 93, 6787. 


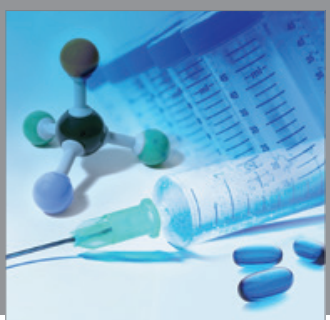

International Journal of

Medicinal Chemistry

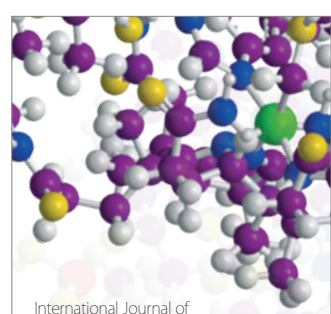

Carbohydrate Chemistry

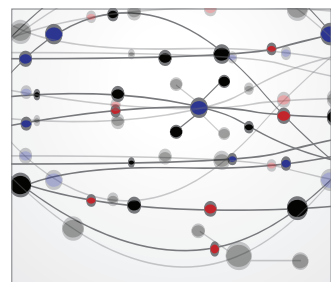

The Scientific World Journal
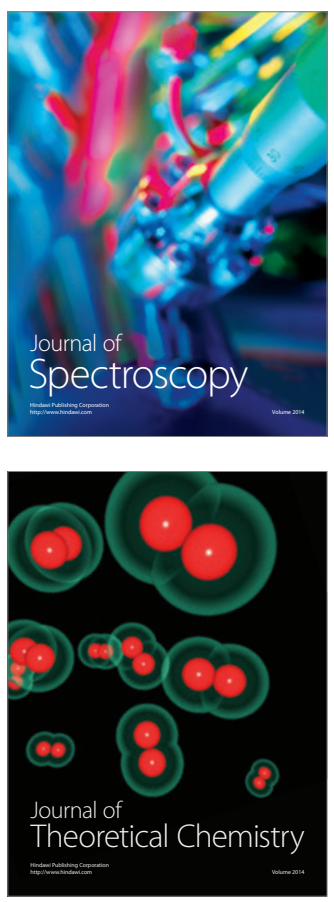
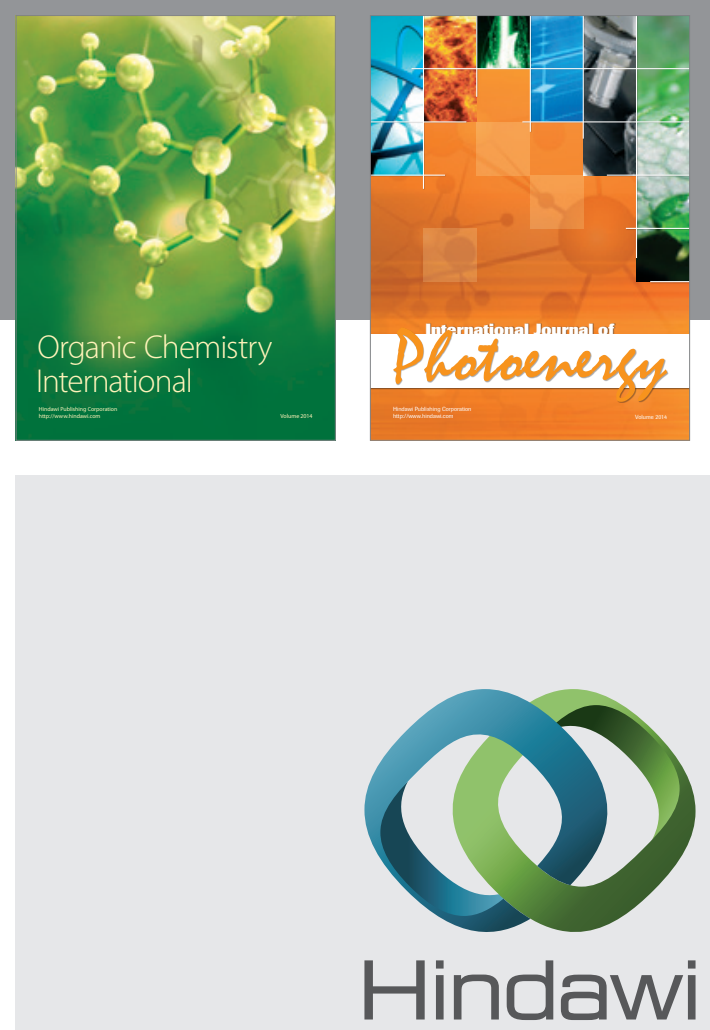

Submit your manuscripts at

http://www.hindawi.com
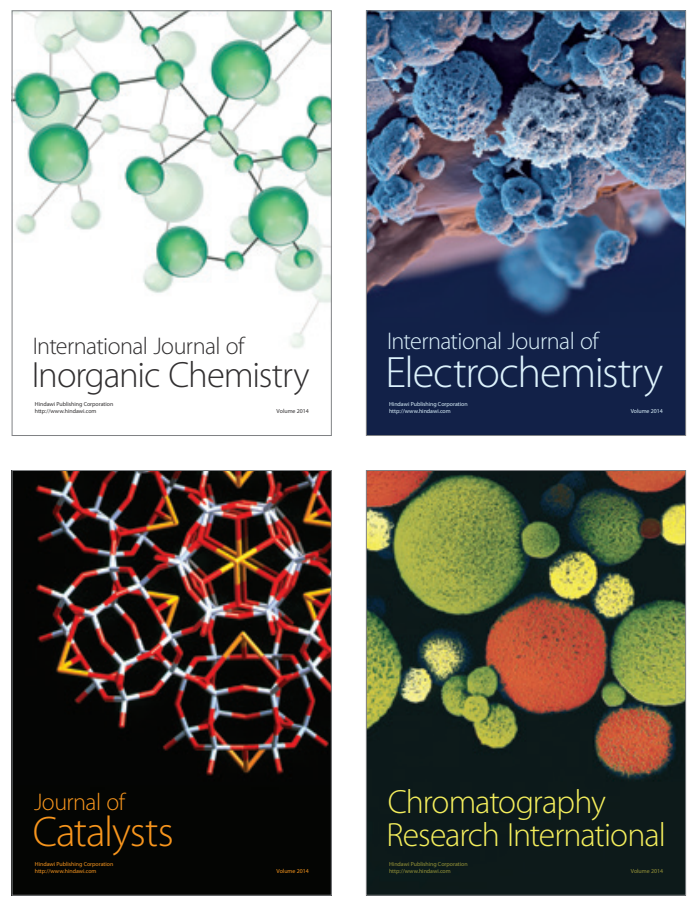
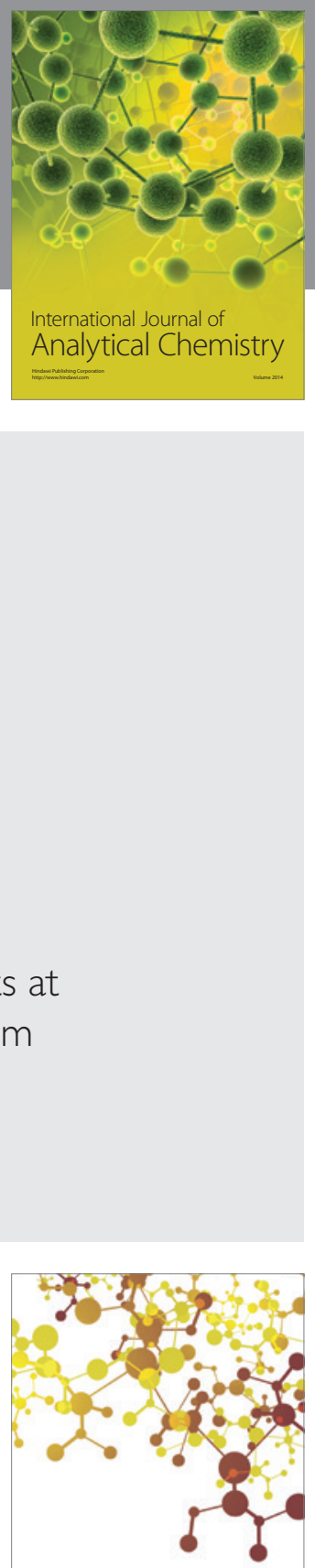

Journal of

Applied Chemistry
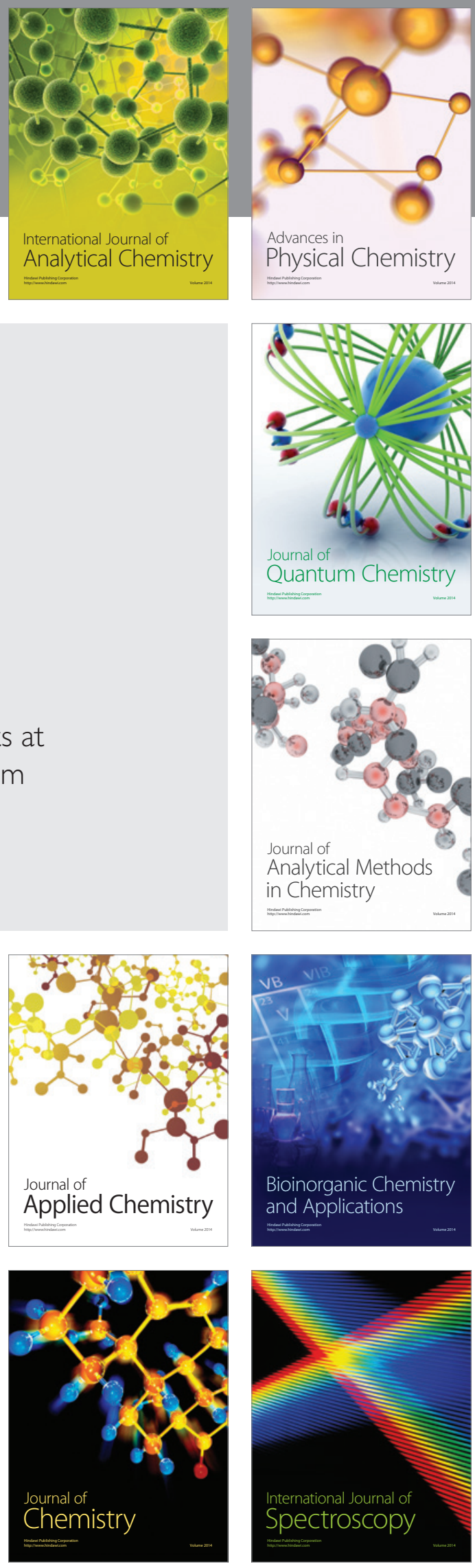